\title{
APRENDIENDO CON TECNOLOGÍAS \\ DE LA INTELIGENCIA EN LA WEB SEMÁNTICA
}

\author{
LEARNING WITH TECHNOLOGY \\ INTELLIGENCE IN THE SEMANTIC WEB
}

Cibertrónica: Aprendiendo con tecnologías de la inteligencia en la web semántica, Díaz de Santos-UNAM, México, 2013. Ruiz-Velasco Sánchez, Enrique

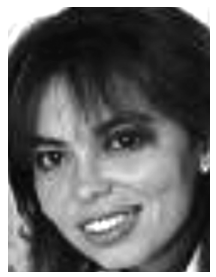

Doctora en Pedagogía, Universidad Nacional Autónoma de México. Licenciada en Ciencias de la Comunicación, Facultad de Ciencias Políticas y Sociales, Universidad Nacional Autónoma de México. Maestra en Ciencias de la Comunicación, Facultad de Ciencias Políticas y Sociales, Universidad Nacional Autónoma de México. Académica de la Facultad de Pedagogía, especializada en temas de Tecnología educativa y Metodología de la investigación.

Correo electrónico: [cortega@up.edu.mx].

\section{INTRODUCCIÓN}

En sus aproximadamente 40 años de existencia, la evolución de Internet ha permitido, por un lado, que los desarrollos tecnológicos relacionados con ésta sean más cercanos a los procesos de enseñanzacomunicación-aprendizaje y, por el otro, que se estén consolidando ideas como la web semántica entendida como el espacio en Internet que se construye en y a través de significados cuyo objetivo es ordenar los contenidos en el ciberespacio.

La web ha pasado por tres etapas claramente definidas: primero fue la web tradicional, en donde los usuarios se ubicaban principalmente como consumidores de información; si recordamos cuando inició la 
web, nos encontrábamos en un entorno estático, con páginas en HTML (HyperText Markup Language) que sufrían pocas actualizaciones y no tenían interacción con el usuario. La web 1.0 es la web tradicional que se caracteriza porque el contenido e información de un portal, sitio o página es producido por un editor especialista en código HTML para luego ser consumido por los visitantes de éste.

Más tarde se da el paso a la web 2.0 como una actitud hacia el aumento de las posibilidades de producir y publicar materiales. Algunos de los fenómenos que lo permiten son: la incorporación de la ideología de software abierto, los recursos de libre acceso y las licencias Creative Commons. El software denominado como abierto se refiere a un programa de cómputo que presenta su código disponible para ser modificado por la comunidad de programadores a nivel mundial, ello permite que se desarrolle y distribuya libremente.

Las licencias Creative Commons (CC) ayudan a conservar los derechos autorales, invitando a usar la obra bajo el esquema de algunos derechos reservados y dando crédito a los autores sobre la misma. Existen ya innumerables espacios en la web que ofrecen las licencias para etiquetar la obra, por ejemplo el espacio para alojar videos como Blip.tv presenta la opción de las distintas versiones de licencias. Para mayor información sobre el tema se sugiere consultar [http://creativecommons.org.mx/que/en], también existen espacios para descargar materiales bajo estas licencias como: [http:/ / search.creativecommons.org/].

Ello permitió el aumento considerable de la interactividad e interacción de los usuarios, ayudando a no ser sólo consumidores sino productores. La anterior idea permite que tecnologías como Internet pasen de ser un almacén de información a un espacio de creación de materiales, factor esencial para el aprendizaje.

Estos tres momentos en la evolución de la web, replantean las prácticas educativas para pensar en una web semántica como un espacio de interacción en donde confluyan los procesos de aprendizaje-comunicación-enseñanza. 


\section{LA WEB SEMÁNTICA}

El fenómeno de la evolución a la web 3.0 o web semántica es relativamente reciente. Aquí se busca organizar la información y el conocimiento que los usuarios han puesto a disposición en la web, a través de diversos desarrollos como los «tesauros» y las «ontologías».

El tesauro es una lista de términos o palabras empleados para representar conceptos, los cuales se formalizan por la comunidad científica y académica, ello permite realizar búsqueda más eficientes, pues la información se ordena a través de los términos acordados previamente. Es importante destacar que dicha lista se actualiza constantemente. Un ejemplo de éstos es el de la UNESCO, el cual se puede consultar en la siguiente liga: [http:/ / databases.unesco.org/thessp/].

En Informática, el término ontología refiere a una estructura conceptual sistematizada y de consenso que almacena, busca y recupera información. Es dentro de la web semántica que las ontologías son una herramienta para el intercambio y uso de conocimientos. Una ontología se forma por una taxonomía relacional de conceptos y por un conjunto de axiomas o reglas de inferencia mediante los cuales se podrá inferir nuevo conocimiento.

Es en este espacio que resulta importante el trabajo con «metadatos», entendidos como el conjunto de datos que describen el contenido de un material, éstos sirven para la clasificación de los materiales y son uno de los temas relevantes de la web 3.0 y estándares de clasificación que ordenen a lo producido y difundido en Internet.

Identificar la importancia de la web semántica a través de las tecnologías de la inteligencia para el aprendizaje plantea varias preguntas: ¿los usuarios utilizan los operadores boléanos para delimitar sus búsquedas?, ¿conocen las bases de datos especializadas para el área de su interés?, ¿poseen criterios para valorar la información que encuentran en la Red?, ¿conocen las posibilidades de la web 3.0 o web semántica como los tesauros? Éstos son algunos elementos a considerar cuando se busca la integración racional de la tecnología a los diversos procesos que confluyen en el aprendizaje. 


\section{CIBERTRÓNICA: APRENDER CON TECNOLOGÍAS DE LA INTELIGENCIA EN LA WEB SEMÁNTICA}

Para ejemplificar un caso específico de aplicación de la web semántica en educación se eligió trabajar sobre la Cibertrónica, concepto innovador de aplicación inteligente de las Tecnologías de la Información, la Comunicación, el Conocimiento y el Aprendizaje (TICCA).

Definiremos a la cibertrónica como la integración racional e inteligente de tecnologías, procesos, métodos, desarrollos o técnicas útiles para abordar de manera inteligente, holista y lúdica el aprendizaje cibertrónico.

Del mismo modo, concebiremos como aprendizaje cibertrónico al aprendizaje significativo, constructivista, construccionista y conectivista que se realiza en y desde el ciberespacio mediante la participación, construcción, cooperación y colaboración entre usuarios que conforman la $w w w$ en distintos niveles y sectores con tecnologías, recursos, ideas, problemas, proyectos, paradigmas, opiniones, necesidades, servicios y aplicaciones (p. 24).

Aparece en la escena la Cibertrónica como estrategia inteligente de integración de la tecnología para el aprendizaje; esto se enmarca en un contexto el cual se sugiere contemple dimensiones estructuradoras de todo proyecto de cómputo académico las cuales se enumeran en el siguiente apartado.

\section{DIMENSIONES ESTRUCTURADORAS DE UN PROYECTO DE CÓMPUTO EDUCATIVO}

Actualmente existe el interés de integrar los procesos que aluden al aprendizaje Tecnología Informáticas (TI) a las instituciones educativas. Esto implica reajustar políticas educativas, infraestructura, contenidos, gestión, modelo de uso y evaluación; dichas dimensiones se ubican en tres espacios importantes que denominamos: meso y macro.

Algunas de dichas dimensiones se trabajan a continuación.

Políticas educativas como parte del meso espacio: 
El objeto de las políticas educativas son los aprendizajes futuros, posibles y deseables de nuestra sociedad. Son resultado de la gestión de tomadores de decisiones para lograr resultados previstos. Una política pública exitosa es aquella que plantea la participación de la inteligencia colectiva. En este sentido, las principales áreas de análisis se enfocan hacia la economía y la infraestructura educativas, así como a los planes de desarrollo anual y sexenal [...] (p. 100).

En lo macro se ubicaría, la infraestructura, los contenidos, la gestión del conocimiento, el modelo de uso y la evaluación:

La infraestructura dará cuenta de los recursos disponibles para la enseñanza aprendizaje: salón, recursos didácticos, computadoras y periféricos, conexiones, conectividad (lograr intercambio de información entre dispositivos sin necesidad de estar conectados), navegadores, bases de datos, programas de aplicación, entre otros (p. 62).

Los contenidos digitales se refieren a los temas y conceptos que favorecen la educación permanente, el diálogo cultural y el desarrollo económico de sus usuarios y serán susceptibles de ser usados, divulgados, compartidos y distribuidos en las comunidades de aprendizaje (p. 76).

Una vez asegurada la infraestructura y un modelo de uso flexible y eficaz, los contenidos serán recursos educativos digitales (considerando el software e Internet) pertinentes y de calidad (p. 73).

En el tema de gestión del conocimiento habría que considerar dos espacios, por un lado la gestión institucional y por el otro la gestión personal. Específicamente, al aludir a esta última, se presenta la siguiente cita:

La gestión alude a las competencias que [se] requieren para enfrentar con efectividad las funciones cognitivas y educativas.

El conocimiento es el más valioso activo individual, organizacional y social en la actualidad. Las formas de gestión cognitiva han evolucionado y constituyen nuevos retos para las instituciones y las personas (p. 99).

$\mathrm{Al}$ atender a la teoría de la complejidad, podemos definir el modelo de uso como la representación de un «holón», éste es un sistema considerado como un todo en sí mismo y como parte de un sistema mayor. Cada sistema puede considerarse un holón, un todo que puede 
identificarse a la vez en parte de algo y a su vez está conformado por partes; por ello está en interrelación con otros elementos del sistema como son: la política (filosofía social), filosofía institucional, infraestructura, gestión del conocimiento y los contenidos.

Los modelos de uso están definidos por el conjunto de teorías pedagógicas y estrategias didácticas que incorporan, y los recursos que utilizan, basados en las tecnologías de la información y la comunicación para el conocimiento. Estos modelos están fundamentados en estrategias contextualizadas, flexibles y susceptibles de replicarse, masificarse y generalizarse (p. 74).

Por último, y no por ello menos importante, está la evaluación, la cual aparece en lo macro con la evaluación institucional — que atiende, entre otros elementos, al planteamiento curricular, el cual expresa los objetivos de la institución y los contenidos- y en lo micro con las propuestas didácticas y las estrategias de trabajo docente para la gestión del aprendizaje. En este espacio, la evaluación se entiende como una corrección de trayectoria en donde el punto de llegada son los objetivos.

El identificar estas dimensiones permite tener parámetros para evaluar proyectos de esta naturaleza y tomar decisiones basadas en el conocimiento de los procesos de cada dimensión.

Además de lo anterior, otro elemento a considerar cuando se trabaja la relación tecnología-aprendizaje es el sustento teórico de la misma el cual se examina en el siguiente apartado.

\section{CONECTIVISMO Y APRENDIZAJE}

Cuando se trabaja con el concepto de aprendizaje siempre se presenta la dificultad de identificar el enfoque teórico que puede atribuírsele, sin embargo, cuando se le relaciona con las tecnologías en Red, se está en posibilidad de analizarlo desde el enfoque conectivista, pues dicho enfoque explica la dinámica cambiante de la circulación de la información como sistema adaptativo complejo. 
El conectivismo es una teoría del aprendizaje para la era digital. El aprendizaje ha cambiado a lo largo de las últimas décadas.

El conectivismo, como teoría, intenta proporcionar una comprensión de cómo aprenden los individuos y las organizaciones. El conectivismo plantea que para aprender de forma efectiva en la sociedad actual se requieren de distintas habilidades personales. El conectivismo propone que una de las principales habilidades clave a desarrollar es la capacidad de realizar conexiones entre ideas, conceptos y campos (p. 41).

A principios del siglo XXI, George Siemens presenta unos postulados teóricos acerca del aprendizaje a partir de la incorporación de las tecnologías informáticas en Red. Dicha teoría se denomina «conectivismo». La concepción conectivista del aprendizaje entiende a éste como la construcción personal que un sujeto hace de un objeto a través de la interacción, dicha construcción pasa necesariamente por procesos interconectados como la información, comunicación y el conocimiento.

Para el conectivismo, entonces, el aprendizaje se vuelve un proceso de conectar nodos o fuentes de información especializada. El aprendizaje y el conocimiento están presentes en la diversidad de opiniones. El aprendizaje personal y organizacional son tareas integradas. El conocimiento personal se compone de una red que se alimenta en las organizaciones e instituciones que, a la vez, alimenta a la red y sigue proporcionando aprendizaje al individuo (p. 42).

El conectivismo es la integración de principios explorados por las teorías de caos, redes, complejidad y auto-organización, y concibe al aprendizaje como conocimiento aplicable enfocado a conectar conjuntos de información especializada.

El conectivismo, como parte de la inteligencia colectiva, busca construir las relaciones pertinentes para encontrar y estructurar el conocimiento que está distribuido a través de una red de conexiones, en este caso, la web (p. 104). 


\section{EPÍLOGO}

A manera de cierre podemos señalar que estas ideas de integración de la tecnología a los espacios educativos dejan abierta la posibilidad a la innovación, entendida como la capacidad de crear productos o ideas que impliquen un progreso; es decir, todo proceso innovador involucra un cambio que debe distinguirse por conllevar beneficios $\mathrm{u}$ oportunidades de mejora.

La clave del éxito en la integración de TI a los procesos de aprendizaje está en asumir la importancia de comunicar, movilizar, desplegar y activar el conocimiento como principio del aprendizaje personal y social.

Claudia Fabiola Ortega Barba 